\title{
100-Year-Old Vaccine for a Novel Virus: Killing Two Birds with One Stone!
}

\section{Introduction}

The coronavirus pandemic has spread like wildfire across the globe. Some countries are more affected than others while children seem to have a milder form of the disease as compared to adults. ${ }^{[1]}$ We are still grappling to understand the reason behind these disparities. There appears to be a correlation between Bacillus Calmette-Guérin (BCG) vaccination and protection against COVID-19. ${ }^{[2,3]}$ Some experts hypothesize that countries offering BCG vaccine in the national immunization program at birth seem to have a lower death rate from COVID-19. ${ }^{[3]}$ Whether this can be attributed to the BCG vaccine or a less virulent virus strain or social distancing measures or because countries like India are just entering phase III of the epidemic will only be known retrospectively. If BCG is indeed the reason for this protective effect, can it be attributed to persistence of "heterologous" or "off-target" effects of the BCG vaccine, will unfold over the next few months. Banking on the "heterologous effect" of BCG, two clinical trials NCT04327206 and NCT04328441 propose to administer prophylactic BCG vaccine to health-care providers in Australia and the Netherlands respectively. At this point in time, there are 3 trials in the Clinical Trials Registry of India using different BCG strains to harness this heterologous effect against COVID-19. The research hypothesis is activation of the innate immune system to protect against progression of the Coronavirus infection. ${ }^{[4]}$ In this article, we try to understand the underlying mechanisms of immune protection, "trained immunity" conferred by the BCG vaccine and review some epidemiological evidence that support or refute the hypothesis.

\section{Background}

The BCG vaccine has been in use since 1921 and about 160 million newborn children receive $B C G$ vaccine annually (2017 data). ${ }^{[5,6]}$ The efficacy of BCG vaccine against pulmonary tuberculosis (TB) is highly variable ranging from $0 \%$ to $80 \%$, but it is highly efficacious against tuberculous meningitis and miliary $\mathrm{TB}$ in children under 5 years of age. ${ }^{[6]}$ BCG offers protection against leprosy and other mycobacteria due to cross-reactive antigens. This effect is called "Cross-Protective effect." "[]] In addition, BCG vaccination has shown to reduce overall pediatric mortality, far more than its target effect on pulmonary TB. This is also true for measles, smallpox, and oral polio vaccine. In addition, concurrent or previous administration of BCG is associated with significantly higher levels of antibodies against hepatitis $\mathrm{B}$, polio, and influenza vaccines ${ }^{[8]}$ Thus BCG vaccine acts as an adjuvant to concurrent or subsequent immunization through its immunostimulatory effect on the innate immune system. Clinically, BCG reduced all-cause mortality in children in several observational studies and randomized trial settings and decreased hospitalization rates due to respiratory infections and sepsis. ${ }^{[5,9-11]}$ These effects of BCG are known as "off-target effect" or "heterologous effect." "[] The heterologous effects of BCG are mediated through the cells of the innate immune system including monocytes/macrophages and natural killer (NK) cells and are independent of memory $\mathrm{T}$ or $\mathrm{B}$ cells. This phenomenon is associated with a memory-like response of the innate immune cells and is known as "trained immunity." ${ }^{[12]}$

\section{Immunology of training the immune system}

Understanding the underlying mechanism of BCG induced immune effects is still a work in progress. Both the innate and the adaptive immune systems mount a response against the bacillus in the BCG vaccine. The effects being both, specific on-target effects, mediated mostly by the $\mathrm{T}$ and $\mathrm{B}$ cells (adaptive immunity), and off-target effects mediated by actors of the innate immune system mainly monocyte/ macrophage system and NK cells. Until recently, it was thought that the innate immune system is the first line of defense and does not have memory. This paradigm has changed recently. Here, we try to understand at least some of the mechanisms of how the bacillus in the vaccine activates the immune system and sustains the effect for a significant period of time called "trained immunity."

Kleinnijenhuis et al. injected healthy volunteers, between 20 and 36 years of age with BCG vaccine. In vitro cytokine assays were performed on peripheral blood mononuclear cells after nonspecific stimulation on day 14 and 3 months later. There was an enhanced Th1 cytokine response (Interleukin [IL] 1- $\beta$, Interferon-gamma [IFN- $\gamma$ ] and Tumor Necrosis Factor- $\alpha$ [TNF- $\alpha]$ ) to nonspecific stimuli such as Staphylococcus aureus and Candida albicans that were significantly enhanced at day 14 and lasted at least 3 months. This was attributed to an increased number and altered functional state of monocytes. The increased cytokine response correlated with increased mRNA expression of these cytokines. This was brought about by long-term transcriptional regulation though histone modification. It was shown that the lysine 4 residue on histone $\mathrm{H} 3$ (H3K4) undergoes trimethylation. H3K4 trimethylation at specific loci leads to increased transcription of proinflammatory cytokine genes. ${ }^{[13]}$

Pattern recognition forms the mainstay of innate immune system. Innate immunity to microbial pathogens relies on the specific detection of pathogen-associated molecular patterns by specific host receptors. Muramyl dipeptide (MDP) is a peptidoglycan, common to cell wall of Gram-positive and Gram-negative bacteria. NOD-2 is an intracellular pattern 
recognition receptor expressed, specifically in monocytes and macrophages. The heterologous immune response to BCG seems to be mediated by the interaction of NOD2 with MDP in the BCG cell wall through activation of the Nuclear factor kappa B (NFkB) pathway. ${ }^{[13]}$

Furthermore, Arts et al. demonstrated that the upregulation of Th1 cytokines after BCG vaccination is dependent on changes in cellular metabolism. They noted an increase in glycolysis, upregulation of glutamine metabolism, and oxidative phosphorylation after stimulation with BCG at day 7 in healthy volunteers. These shifts in metabolism are dependent on the mammalian target of rapamycin (mTOR) pathway and histone methylation and persisted even at 3 months post vaccination. These metabolic and epigenetic modifications are intertwined and necessary for development of trained immunity. ${ }^{[14]}$

In the same cohort of healthy volunteers studied by Kleinnijenhuis et al., ${ }^{[13]}$ administration of BCG vaccine led to increased production of proinflammatory cytokines upon stimulation with heat inactivated $C$. albicans at 3 months after vaccination. It was also seen that in severe combined immunodeficiency mice with deficient $\mathrm{T}$ and $\mathrm{B}$ lymphocytes but preserved NK cell lineage, $\mathrm{BCG}$ vaccination-induced protection against disseminated Candida Albicans infection, establishing the protective effect of NK cells. ${ }^{[15]}$ This NK cell-mediated cytotoxic effect is exploited effectively in the treatment of bladder cancers. Intravesicular instillation of BCG is an effective treatment option in superficial bladder cancers. ${ }^{[16]}$

The non-specific effects of the BCG vaccine are also effective against viruses. In another study (NCT02114255) by the same group at the Nijmegen Medical Center, healthy volunteers were randomized to receive $\mathrm{BCG}$ vaccine $(n=20)$ or placebo $(n=20) 14$ days prior to trivalent influenza vaccine. In the BCG-vaccinated group, hemagglutination-inhibiting antibody responses against 2009 pandemic $\mathrm{H} 1 \mathrm{~N} 1$ vaccine strain were significantly enhanced with a trend toward rapid seroconversion defined as $>4$-fold increase in titers as compared to baseline. ${ }^{[17]}$ In an experiment by Floc'h et al., mice were inoculated with Pasteur Institute BCG and challenged with different viruses introduced by various routes 15-31 days later. BCG-inoculated mice exhibited a significantly higher resistance to these lethal infections than control mice (overall survival $18 \%$ vs. $41 \%$ in BCG inoculated mice). ${ }^{[18]}$ Thus, BCG also acts as an adjuvant to potentiate responses to a viral challenge, possibly viral infections like coronavirus.

The off-target or heterologous effects of the BCG vaccine are thus mediated by the monocyte/macrophages and NK cells of the innate immune system. Now, we understand that these effects can last longer through epigenetic changes that go hand-in-hand with metabolic shifts in these cells. ${ }^{[13,14]}$ However, how long can these changes persist, what is their clinical relevance and how can they be exploited in our fight against a novel virus? Is there any evidence?

\section{Epidemiological evidence for trained immunity}

In 1927, Carl Näslund was first to note that BCG vaccination at birth had a mortality almost threefold lower than the very high rate of $10 \%$ among unvaccinated children. ${ }^{[19]}$ A systematic analysis of several observational studies and clinical trials by the WHO (Higgins et al.) has shown reduction in overall mortality by more than expected through their on-target effects. The strongest effect was seen in the first 28 days and in premature infant. ${ }^{[9]}$ BCG contributed to an overall $30 \%$ reduction in all-cause neonatal mortality (95\% confidence interval $[\mathrm{CI}]: 1 \%-51 \%)$. In the two randomized control studies with minimum bias, the reduction in neonatal mortality was about $48 \%(95 \% \mathrm{CI}$ : $18 \%-67 \%)$. $^{[20,21]}$ In contrast. a study done by Jayaraman et al. that reported results of two trials in India did not show any protective effect on neonatal mortality. This dichotomy could also be related to nutritional status of the mother and child, immunization status of the mother, host genetics, and access to health care. This could also be related to the genetic variation in BCG strain, although there is no such direct evidence to prove so. ${ }^{[2]}$ Where BCG is protective, the duration of protection is variable with an average of about 10 years. Approximately 50\% protection has been observed 15-20 years after vaccination in Brazil and 40-50 years after vaccination in American Indians and Alaskan natives. It is hypothesized that BCG vaccination has higher efficacy in more temperate areas, probably related to lower exposure to nontuberculous mycobacteria (NTM). ${ }^{[23]}$ Furthermore, the effects of environmental NTM can precede BCG vaccination and block multiplication of the vaccine strain. ${ }^{[24]}$ Therefore, to suggest that BCG at birth might offer protective immunity against SARS-CoV-2 is preposterous to assume.

\section{Discussion}

Considering that BCG has heterologous effects that can last for a longer duration of time sets a persuasive hypothesis that the vaccine might offer some protection from severe infection from SARS-CoV-2. There are epidemiological data to suggest that $\mathrm{BCG}$ offered protection from acute respiratory illnesses in addition to bacterial infections. ${ }^{[1]}$ However, given that the efficacy is highly variable from $0 \%$ to $80 \%$ and wanes over time, it might be premature to say that the low death rate due to coronavirus pandemic in certain countries is related to the protective effect of $\mathrm{BCG}$ at birth.

The NCT04327206 clinical trial is a good idea as it is trying to activate the innate immune system in preparation to face the virus. Does re-vaccination work? Again, the jury is still out there. Retrospective observation in Hungary and Poland, in 1950s-60s, showed reduced incidence of TB after re-vaccination. In Chile, where re-vaccination was given at 6 years and 14 years; there was no difference in the proportion of young adults with 1,2 , or 3 BCG scars. ${ }^{[25]}$ In a randomized control trial in South Africa, where incidence of TB was high, re-vaccination seemed 
to offer sustained reconversion of IFN- $\gamma$ release after BCG re-vaccination. This effect was measured at day 70 and up to 2 years after re-vaccination. ${ }^{[26]}$ The off-target effects and clinical impact on non-TB infections were not evaluated in these trials. ${ }^{[27]}$ In a similar prospective randomized trial in South India, IFN- $\gamma$ and IL-2 and TB antigen-specific CD4 and CD8 T cell responses were boosted at 4 and 34 weeks after re-vaccination. In addition, innate IFN- $\gamma$, NK cell, $\gamma \delta \mathrm{T}$ cell, and NKT cell responses were higher in re-vaccinated persons as compared to placebo controls. The TB antigen-specific innate immunity peaked transiently at week 4, while BCG specific peak was seen later. ${ }^{[28]}$ One of the contentions of using BCG re-vaccination is the duration of onset of nonspecific immunity is longer. If we look at antigen-specific immunity as in the Indian trial, the peak was seen at 4 weeks. In the epigenetic modulation studies by the Dutch group, non-specific immune responses were seen at day 14 post-vaccination. ${ }^{[13]}$ The trained immune responses lasted for about 3 months to 1 year in different trial settings. ${ }^{[26,28]}$

In the Guinea Bissau study for low birth weight infants, a protection from infections was seen as early as first 3 days of life after BCG vaccination at birth. Therefore, a 14-day window might be sufficient to rev up the innate immune system as the front line of defense against the virus.

Given the heterogeneity of the data on BCG in India, well-designed clinical trials in the Indian setting will target two birds in the same stone, the virus and the mycobacteria.

\section{Chetan Dhamne1, Gagan Prakash², Gaurav Narula ${ }^{1}$, Shripad Banavali ${ }^{1}$, Jyoti Bajpai ${ }^{1}$}

${ }^{I}$ Department of Medical Oncology, TMC, Mumbai, Maharashtra, India, ${ }^{2}$ Department of Surgical Oncology, TMC, Mumbai, Maharashtra, India

Address for correspondence: Dr. Chetan Dhamne, Department of Medical Oncology, TMC, Mumbai, Maharashtra, India. E-mail: chetandhamne@gmail.com

Submitted: 15 -Apr-2020 Accepted in Revised From: 12-May-2020 Published: 27-Jun-2020

\section{References}

1. Dong Y, Mo X, Hu Y, Qi X, Jiang F, Jiang Z, et al. Epidemiological characteristics of 2143 pediatric patients with 2019 coronavirus disease in China. Pediatrics April 2020:e20200702. DOI: 10.1542/peds.2020-0702.

2. Miller A, Reandelar MJ, Fasciglione K, Roumenova V, Li Y, Otazu G. Correlation between universal BCG vaccination policy and reduced morbidity and mortality for COVID-19: An epidemiological study. medRxiv 2020.03.24.20042937.

3. Hegarty P, Sfakianos J, Giannarini G, DiNardo A and Kamat A. Covid-19 and Bacillus Calmette-Guerin: What is the Link? Eur Urol Oncol 2020. DOI 10.1016/j.euo.2020.04.001.

4. Available from: https://clinicaltrials.gov/ct2/results?term=BCG\& cond=COVID-19. [Last accessed 2020 May 07].

5. Curtis N. BCG vaccination and all-cause neonatal mortality. Pediatr Infect Dis J 2019;38:195-7.
6. Trunz BB, Fine $P$, Dye $C$. Effect of $B C G$ vaccination on childhood tuberculous meningitis and miliary tuberculosis worldwide: A meta-analysis and assessment of cost-effectiveness. Lancet 2006;367:1173-80.

7. Zimmermann P, Finn A, Curtis N. Does BCG vaccination protect against nontuberculous mycobacterial infection? A systematic review and meta-analysis. J Infect Dis 2018;218:679-87.

8. Zimmermann $\mathrm{P}$, Curtis $\mathrm{N}$. The influence of $\mathrm{BCG}$ on vaccine responses - A systematic review. Expert Review of vaccines 2018;17:547-54.

9. Higgins JP, Soares-Weiser K, López-López JA, Kakourou A, Chaplin K, Christensen H, et al. Association of BCG, DTP, and measles containing vaccines with childhood mortality: Systematic review. BMJ 2016;355:i5170.

10. Hollm-Delgado MG, Stuart EA, Black RE. Acute lower respiratory infection among Bacille Calmette-Guérin (BCG)-vaccinated children. Pediatrics 2014;133:e73-81.

11. de Castro MJ, Pardo-Seco J, Martinón-Torres F. Nonspecific (Heterologous) Protection of Neonatal BCG Vaccination Against Hospitalization Due to Respiratory Infection and Sepsis. Clin Infect Dis 2015;60:1611-9.

12. Netea MG, Quintin J, van der Meer JW. Trained immunity: A memory for innate host defense. Cell Host Microbe 2011;9:355-61.

13. Kleinnijenhuis J, Quintin J, Preijers F, Joosten L, Ifrim D, Saeed S, et al. Bacille Calmette-Guerin induces NOD2dependent nonspecific protection from reinfection via epigenetic reprogramming of monocytes. PNAS 2012;109:1753742.

14. Arts RJ, Carvalho A, La Rocca C, Palma C, Rodrigues F, Silvestre R, et al. Immunometabolic pathways in BCG-induced trained immunity. Cell Rep 2016;17:2562-71.

15. Kleinnijenhuis J, Quintin J, Preijers F, Joosten LA, Jacobs C, Xavier RJ, et al. BCG-induced trained immunity in NK cells: Role for non-specific protection to infection. Clin Immunol 2014;155:213-9.

16. Brandau S, Suttmann H, Riemensberger, Seitzer U, Arnold J, Durek C, et al. Perforinmediated lysis of tumor cells by mycobacterium bovis bacillus calmette guerin activated killer cells. Clin Can Res 2000;6:372938.

17. Leentjens J, Kox M, Stokman R, Gerretsen J, Diavatopoulos DA, van Crevel $\mathrm{R}$, et al. BCG Vaccination Enhances the Immunogenicity of Subsequent Influenza Vaccination in Healthy Volunteers: A Randomized, Placebo-Controlled Pilot Study. J Infect Dis 2015;212:1930-8.

18. Floc'h $\mathrm{F}$ and Werner $\mathrm{G}$. Increased resistance to virus infections of mice inoculated with BCG (Bacillus Calmetteguerin) Ann Immunol 1976;127:17386.

19. Aaby P, Benn CS. Saving lives by training innate immunity with bacilli Calmette-Guerin vaccine. Proc Natl Acad Sci U S A 2012; 109:17317-8.

20. Aaby P, Roth A, Ravn H, Napirna BM, Rodrigues A, Lisse IM, et al. Randomized trial of BCG vaccination at birth to low-birth-weight children: Beneficial nonspecific effects in the neonatal period? J Infect Dis 2011;204:245-52.

21. Biering-Sørensen S, Aaby P, Napirna BM, Roth A, Ravn H, Rodrigues A, et al. Small randomized trial among low-birth-weight children receiving bacillus Calmette-Guérin vaccination at first health center contact. Pediatr Infect Dis J 2012;31:306-8.

22. Schaible UE, Linnemann L, Redinger N, Patin EC, Dallenga T. Strategies to Improve Vaccine Efficacy against Tuberculosis by Targeting Innate Immunity. Front Immunol 2017;8:1755.

23. Dye C. Making wider use of the world's most widely used vaccinie: Bacille Calmette-Guerin revaccination reconsidered. 
J Royal Soc Interf 2013; Interface.10:20130365.

24. Dockrell HM, Smith SG. What have we learnt about BCG vaccination in the last 20 years? Front Immunol 2017;8:1134.

25. WHO statement on BCG revaccination for the prevention of tuberculosis. Bulletin of the World Health Organization 1995; 73:805-10.

26. Nemes E, Geldenhuys H, Rozot V, Rutkowski KT, Ratangee F, Bilek N, et al. Prevention of M. tuberculosis infection with H4:IC31 vaccine or BCG revaccination. $N$ Engl J Med 2018;379:138-49.

27. de Bree C, van Crevel R, Netea MG. H4:IC31 vaccine or BCG revaccination for tuberculosis. N Engl J Med 2018;379:1969.

28. Rakshit S, Ahmed A, Adiga V, Sundararaj BK, Sahoo PN, Kenneth J, et al. BCG revaccination boosts adaptive polyfunctional Th1/Th17 and innate effectors in IGRA+and IGRA Indian adults. JCI Insight 2019;4:e130540.
This is an open access journal, and articles are distributed under the terms of the Creative Commons Attribution-NonCommercial-ShareAlike 4.0 License, which allows others to remix, tweak, and build upon the work non-commercially, as long as appropriate credit is given and the new creations are licensed under the identical terms.

\begin{tabular}{|l|l|}
\hline \multicolumn{2}{|c|}{ Access this article online } \\
\hline Quick Response Code: & Website: \\
& www.ijmpo.org \\
\cline { 2 - 2 } & DOI: \\
\hline
\end{tabular}

How to cite this article: Dhamne C, Prakash G, Narula G, Banavali S, Bajpai J. 100-year-old vaccine for a novel virus: Killing two birds with one stone! Indian J Med Paediatr Oncol 2020;41:291-4. 